\title{
Formal concepts expressed by compositional hierarchical Hebbian cell assemblies Thomas Wennekers
}

\author{
Address: Centre for Theoretical and Computational Neuroscience, University of Plymouth, PL4 8AA, Plymouth, UK \\ Email: Thomas Wennekers - thomas.wennekers@plymouth.ac.uk
}

from Eighteenth Annual Computational Neuroscience Meeting: CNS*2009

Berlin, Germany. 18-23 July 2009

Published: 13 July 2009

BMC Neuroscience 2009, I0(SuppI I):P9| doi:|0.II86/I47|-2202-I0-SI-P9|

This abstract is available from: http://www.biomedcentral.com/I47I-2202/I0/SI/P9I

(C) 2009 Wennekers; licensee BioMed Central Ltd.

Hebbian cell assemblies can be formalised as sets of tightly connected cells in auto- and hetero-associative memories. Direct evidence for such "cliques" has recently been obtained in multiple-unit recordings from rat hippocampal neurons $[1,2]$. These experiments suggest a hierarchical organisation where cliques are embedded in each other such that larger cliques represent less specific stimulus conditions. We here suggest an extended interpretation stating that the firing patterns may not just reflect nested categories but a lattice of concepts about stimulus-response mappings in the sense of formal concept analysis, an applied branch of set theory. We present an implementation of formal concept lattices in bidirectional associative memories that, in contrast to previous work by Belohlávek [3], satisfies Dale's principle and uses balanced excitation and inhibition [4]. Inhibitory cells have fixed, non-plastic synapses even if the model learns new concepts. As an extreme case a single global inhibitory cell is enough that controls the total level of activation. The excitatory cells can further learn incrementally using a Hebbian coincidence learning rule. A spiking neuron variant of the model is further presented and implications of the model for retrieval in auto-associative memories are outlined. Overall the model is well suited for representing hierarchical compositional relationships between entities in the form of correlated memory traces in the brain.

\section{Acknowledgements}

The author acknowledges support by the Engineering and Physical Sciences Research Council of the United Kingdom (EP/COI084I/I, COLAMN - A Novel Computing Architecture for Cognitive Systems based on the Laminar Microcircuitry of the Neocortex) and the European Community (IST200I-35282, FACETS - Fast Analog Computing with Emergent Transient States).

\section{References}

I. Lin L, Osan R, Tsien J-Z: Organizing principles of real-time memory encoding: neural clique assemblies and universal neural codes. Trends in Neurosciences 2006, 29:48-57.

2. Harris KD, Csicsvari J, Hirase H, Dragoi G, Buzsaki G: Organization of cell assemblies in the hippocampus. Nature 2003, 424:552-555.

3. Belohlávek R: Representation of concept lattices by bidirectional associative memories. Neural Computation 2000, I 2:2279-2290.

4. Wennekers $\mathrm{T}$ : On the natural hierarchical composition of cliques in cell assemblies. Journal of Cognitive Computation 2009 in press. 